\title{
COVID-19 and gastrointestinal symptoms in Mexico, a systematic review: does location matter?
}

Antonio Pizuorno ${ }^{1}$, Nora A. Fierro², Edgar D. Copado-Villagrana ${ }^{3}$, María E. Herrera-Solís ${ }^{3}$, Gholamreza Oskrochi ${ }^{4}$, Hassan Brim ${ }^{5}$ and Hassan Ashktorab ${ }^{5^{*}}$ (i)

\begin{abstract}
Background: Covid-19 in Mexico is on the rise in different parts of the country. We aimed to study the symptoms and comorbidities that associate with this pandemic in 3 different regions of Mexico.

Methods: We analyzed data from SARS-CoV-2 positive patients evaluated at healthcare centers and hospitals of Mexico $(n=1607)$ including Northwest Mexico (Sinaloa state), Southeast Mexico (Veracruz state) and West Mexico (Jalisco state) between March 1 and July 30, 2020. Mexico consists of a total population that exceeds 128 million. Demographics, comorbidities and clinical symptoms were collected. Statistical descriptive analysis and correlation analyses of symptoms, comorbidities and mortality were performed.

Results: A total of 1607 hospitalized patients positive for COVID-19 across all 3 regions of Mexico were included. The average age was 54.6 years and $60.4 \%$ were male. A mortality rate of $33.1 \%$ was observed. The most common comorbidities were hypertension (43.2\%), obesity (30.3\%) and diabetes (31.4\%). Hypertension was more frequent in West (45\%), followed by Northwest (37\%) and Southeast Mexico (29\%). Obesity was around 30\% in Northwest and West whereas an $18 \%$ was reported in Southeast. Diabetes was most common in West (34\%) followed by Northwest (22\%) and Southeast (13\%). This might be related to the highest mortality rate in Northwest (31\%) and West (37\%) when compared to Southeast. Most common symptoms in our overall cohort were fever (80.8\%), cough (79.8\%), headache (66\%), dyspnea (71.1\%), myalgia (53.8\%), joints pain (50.8\%) and odynophagia (34.8\%). Diarrhea was the main gastrointestinal (Gl) symptom (21.3\%), followed by abdominal pain (18\%), and nausea/ vomiting (4.5\%). Diarrhea and abdominal pain were more common in West (23.1 and 21\%), followed by Southeast (17.8, and 9.8\%) and Northwest (11.4 and 3.1\%).

Conclusion: Our study showed a high mortality rate likely related to high frequencies of comorbidities (hypertension, obesity and diabetes). Mortality was different across regions. These discrepancies might be related to the differences in the frequencies of comorbidities, and partially attributed to differences in socio-economic conditions and quality of care. Thus, our findings stress the need for improved strategies to get better outcomes in our population.
\end{abstract}

Keywords: Coronavirus disease-19, Pandemic, Gastrointestinal manifestation, Diarrhea, Mexico

\footnotetext{
* Correspondence: hashktorab@howard.edu

${ }^{5}$ Department of Medicine, Department of Pathology and Cancer Center, Department of Biochemistry \& Molecular Biology, Howard University College of Medicine, 2041 Georgia Avenue, N.W, Washington, D.C 20060, USA

Full list of author information is available at the end of the article
} 


\section{Background}

After 7 months of SARS-CoV-2 pandemic, Latin America (LA) is the current epicenter with $8,815,850$ diagnosed cases and 325,848 reported deaths as of Sep. 22nd, 2020 [1]. Mexico, the 10th most populated country, with a high poverty rate, major inequalities, and a high prevalence of chronic and metabolic diseases [2], is expected to be one of the most affected countries during the pandemic. According to the evolving data compiled by Johns Hopkins University [3], Mexico has reported more than 700,000 cases and 73,000 deaths as of Sep. 22nd, 2020.

COVID-19 presents primarily as a lower respiratory tract infection, but the multisystemic nature of the disease is commonly found in severe cases. Indeed, a broad spectrum of symptoms associated with COVID-19 has been identified, which range from mild, to moderate to severe symptoms associated with critical illness resulting in respiratory failure or multiorgan dysfunction and/or death. Currently, fever and cough remain the most prevalent symptoms in adults infected by SARS-CoV-2 [4]. Neurological and gastrointestinal symptoms are also encountered [5]. The underlying causes of the variability of COVID-19-related symptomatology and their potential association to different outcomes have not been defined yet. Studies have been conducted in Asian and European populations, the first regions affected by the pandemic. However, differences associated to specific populations present issues with regard to generalizing findings. Evidence supports that human host genetics contribute to the onset of several chronic diseases, including those of infectious nature [6]. This is particularly important in populations with heterogenic heritage such as Mexico, which has an admixture genome [7] differentially distributed across the country [8]. Thus, the study of possible differences in the distribution of COVID-19related symptoms and comorbidities in distinct regions of Mexico and their effect on disease outcomes need to be evaluated.

Herein, we compared the frequency of COVID-19 related symptoms and comorbidities in patients from 3 different regions of Mexico: Culiacan city at the state of Sinaloa representing Northwest, the state of Jalisco representing West and Veracruz city at the state of Veracruz representing Southeast.

\section{Methods}

\section{Search Strategy \& Selection Criteria}

A systematic literature search was carried out of published articles using electronic databases such as PubMed, OVID, Scopus and Google Scholar from March 1st through Jul. 30th, 2020. The following terms were included in the search bar: COVID-19 Mexico.
Also, data from the Ministry of Health of Mexico [9] were retrieved.

Inclusion criteria: The following inclusion criteria were used: Confirmed diagnosis of COVID-19 by RT-PCR as reported by the Ministry of Health of Mexico and crosschecked via Johns Hopkins University COVID-19 dashboard. There was no distinction regarding the number of diagnosed cases, sex, age, treatment and/or outcome. Only hospitalized patients were included in this study. Exclusion criteria: Studies where the cases were not confirmed by RT-PCR. studies with incomplete symptoms or comorbidities reports and studies in outpatient settings.

\section{Study selection}

Using the inclusion and exclusion criteria, two papers Villagran-Olivas (ref 10) and Remes-Troche (ref 11) were retained and their detailed patients' data were retrieved. These two studies had 192 and 112 COVID patients, respectively.

A third set of data that consists of a retrospective study was also included. We analyzed databases of 1303 SARS-CoV-2 RT-PCR confirmed patients hospitalized at the hospitals of the Delegation of the Instituto Mexicano del Seguro Social (IMSS) Jalisco between March 1st and July 30, 2020. Patients with a confirmatory SARS-CoV-2 test were hospitalized at IMSS based on the CURB-65 scale (confusion, urea, respiratory rate, blood pressure and over 65 years age). Initially, if the patients were COVID-19 positive, and had dyspnea or chest pain, vital signs are immediately taken and if saturation is less than $90 \%$, the patient is hospitalized. The study protocol was approved by the Ethics Committee of the IMSS, Jalisco IRB: 2020-1306-104 (Fig. 1).

\section{Regions included in Mexico \\ Northwest Mexico}

Data from 192 SARS-CoV-2 positive hospitalized patients previously reported [10] admitted between Mar. 1st to May 31st, 2020 at the Hospital Civil de Culiacan, a public hospital located in Culiacan city in the Sinaloa state, were retrospectively analyzed. Culiacan city covers $65 \mathrm{~km}^{2}$ (25 sq. mi) and is located at $24^{\circ} 48^{\prime} 25^{\prime \prime} \mathrm{N} 107^{\circ} 23^{\prime}$ $38^{\prime \prime} \mathrm{W}$. It has an elevation of $71 \mathrm{~m}$ above sea level (233 $\mathrm{ft}$ ) and has mean temperature ranges of $70.3^{\circ} \mathrm{F}, 74.5^{\circ} \mathrm{F}$ and $79.5^{\circ} \mathrm{F}$ in the months of March, April and May. The population for the city is 858,638 people ( 166.8 inhab/ $\mathrm{km}^{2}$ ).

\section{Southeast Mexico}

Data from 112 SARS-CoV-2 positive hospitalized patients previously reported [11] admitted between Apr. 1st and May 5th, 2020 at the Hospital Español de Veracruz, a private hospital located in Veracruz city at the 


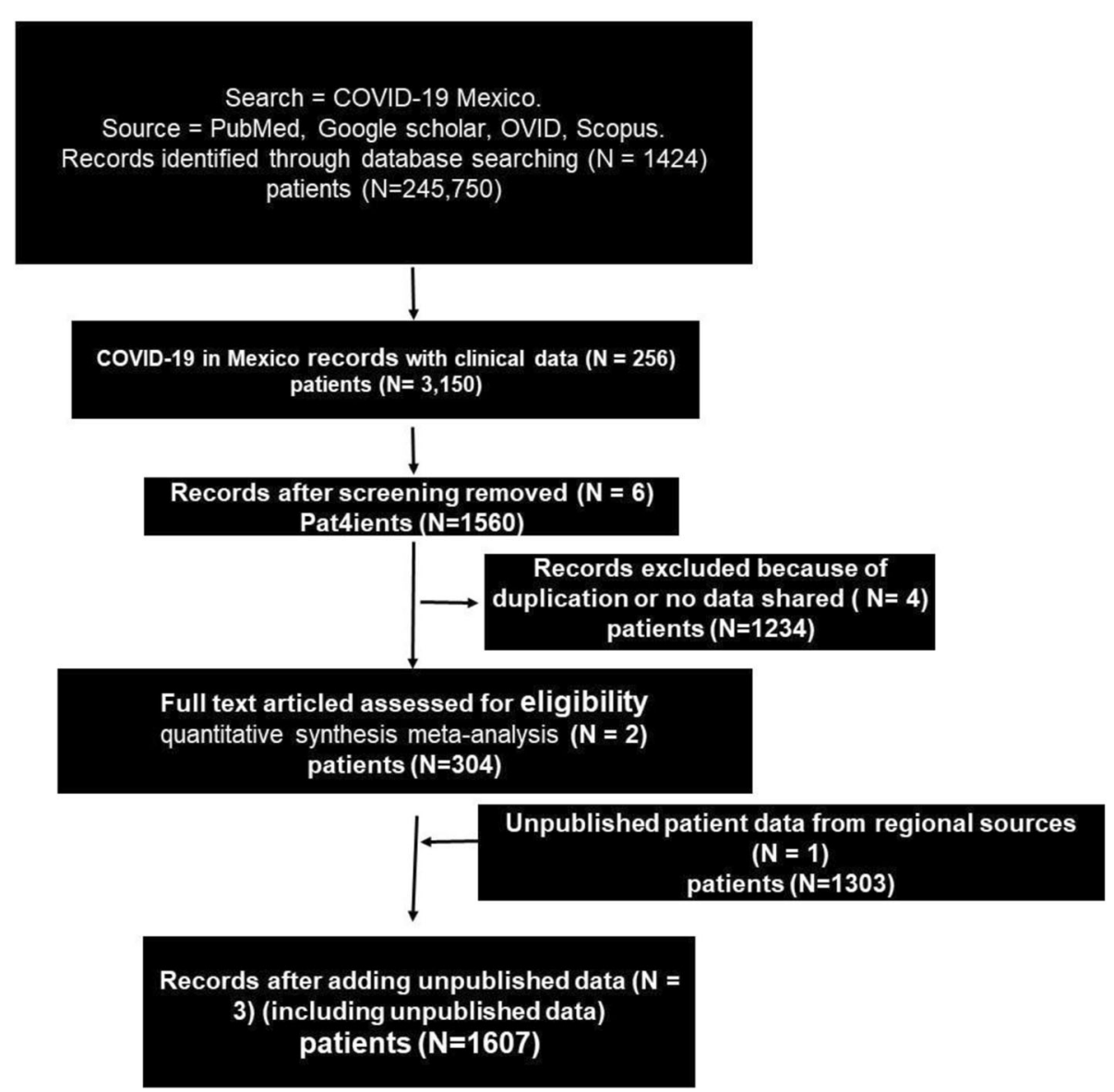

Fig. 1 Fellow chart of the study

Veracruz state, were retrospectively analyzed. Veracruz city covers $90 \mathrm{~km}^{2}$ (56 sq. mi), is located at $19^{\circ} 11^{\prime} 25^{\prime \prime} \mathrm{N}$ $96^{\circ} 09^{\prime} 12^{\prime \prime} \mathrm{W}$, has an elevation of $10 \mathrm{~m}$ above sea level $(30 \mathrm{ft})$ and has mean temperature ranges of $78.4^{\circ} \mathrm{F}$ and $82^{\circ} \mathrm{F}$ in the months of April and May, respectively. The population for the city is 609,829 people.

\section{West Mexico}

A retrospective study was carried out by analyzing databases from West Mexico patients [9] with RT-PCR confirmed SARS-CoV-2 infections of nasopharyngeal swabs. This led to 1303 SARS-CoV-2 patients admitted between Mar. 20th and May 31st, 2020 at the hospitals of the Delegation of the Instituto Mexicano del Seguro Social (IMSS) Jalisco state. The IMSS Jalisco delegation includes 170 medical units, of which 16 are secondary level health care centers and 3 are tertiary level health care centers. The IMSS serves to state formal workers. The state of Jalisco covers $78,599 \mathrm{~km}^{2}$ (30,347 sq. mi), is located at $20^{\circ} 34^{\prime} \mathrm{N} 103^{\circ} 41^{\prime} \mathrm{W}$, and has an altitude which varies from 0 to $4300 \mathrm{~m}$ (o to $14,110 \mathrm{ft}$ ). The mean temperature in various regions of the state ranges from 50 to $66.4{ }^{\circ} \mathrm{F}$ and in other areas from 66.4 to $71.6{ }^{\circ} \mathrm{F}$.
March, April and May are dry months. The population for the state is $7,844,830\left(100\right.$ inhab $\left./ \mathrm{km}^{2}\right)$.

\section{Systematic analysis}

Tables were generated from the retrieved data. The tables included the following information: report date, location, confirmed cases, deaths, median age, symptoms (headache, cough, myalgia, fever, odynophagia, anosmia, dyspnea, ageusia, thoracic pain, tachypnea, cyanosis, nasal congestion, joint pain and fatigue, diarrhea, abdominal pain, nausea and vomiting), comorbidities (hypertension, diabetes, asthma, obesity, cardiovascular disease, COPD, immunosuppression, cancer, IBD). Body Mass Index (BMI) was calculated based on a person's weight in kilograms divided by the square of height in meters. Any person with BMI more than $25 \mathrm{Kg} / \mathrm{M} 2$ and less than 30 is overweight and those $>30$ are considered obese. This study used the American Diabetes Association 2020 Criteria for Diabetes Mellitus diagnosis, patients with a $\mathrm{A} 1 \mathrm{C}$ higher or equal to $6.5 \%$, Fasting plasma glucose higher or equal to $126 \mathrm{mg} / \mathrm{dl}$ are considered diabetic. Our cohort database only included 
patients medical histories (diabetes, hypertension,..) but no medication history.

\section{Statistical analysis}

Patient demographics, symptoms, underlying comorbidities and mortality rates were compared in hospitalized patients from Northwest, West and Southeast Mexico. The common symptoms and comorbidities were combined and analyzed by weighted analysis methods where applicable. Correlation coefficients were calculated, where applicable, to establish associations between comorbidities and mortality. Mortality, also known as Case Fatality Rate correspond to total of dead COVID-19 patients/total COVID-19 patients $(\times 100)$. The effect of symptoms was reported using weighted analysis where weights were related to the size of the reported study. SPSS version 26 (SPSS Inc., Chicago, IL, USA) was used for this analysis.

\section{Results}

\section{High mortality in hospitalized patients in Mexico}

To determine if COVID-19 outcome in Mexico was as reported worldwide, the study population from Northwest, West and Southeast regions was analyzed. A total of 1607 confirmed hospitalized cases were included. According to the overall weighted average analysis, the average age was 54.6 years $( \pm$ SD 3.4_) and the percentage of distribution between males and females was $60.4 \%$ versus $39.4 \%$, respectively. A high mortality rate of $33.1 \%$ was observed (Table 1 ). The mortality rate for hospitalized and ambulatory cases reported by the Ministry of Health in Mexico for the same time period was $10-12 \%[9]$.

\section{Comorbidities in hospitalized COVID-19 Mexican patients} The most common comorbidities in our cohort were hypertension with $43.2 \%$, followed by obesity with $30.2 \%$ and diabetes with $31.4 \%$ (Fig. 2). As for mortality rate, the prevalence of these comorbidities was higher than previously reported by Giannouchos et al. (I think we should eliminate this and refrain to make any discussion and/or comparison of the results with other references in the results section). in a large cohort of 87,756 COVID-19 patients from Mexico (20.5\% for hypertension, $20.9 \%$ for obesity and $17.5 \%$ for diabetes) [12]. These differences might be related to the fact that the above-mentioned report includes hospitalized and ambulatory patients in its analysis and only hospitalized patients were included in our study.

Comorbidities and mortality in hospitalized patients vary in northwest, west and Southeast Mexico

We conducted an analysis to characterize plausible differences in COVID-19 behavior in Northwest relative to
Table 1 Demographic characteristics, mortality, symptoms and comorbidities in hospitalized COVID-19 Mexican patients

\begin{tabular}{ll}
\hline Characteristics & Percentage \\
\hline Patient $n=1607$ & $\%$ \\
Gender (male) & 60.4 \\
Mortality & 33.1 \\
Symptoms & \\
Fever & 80.8 \\
Cough & 79.8 \\
Headache & 66 \\
Fatigue & 56.2 \\
Dyspnea & 71.1 \\
Myalgias & 53.8 \\
Joint pain & 50.8 \\
Odynophagia & 34.8 \\
Nasal congestion & 10.4 \\
Tachypnea & 8.6 \\
Thoracic pain & 33.2 \\
Anosmia & 5.9 \\
Ageusia & 6.1 \\
Cyanosis & 6.7 \\
Hypertension & 43.2 \\
Obesity & 30.3 \\
Diabetes & 31.4 \\
COPD & 6.5 \\
CV disease & 4.4 \\
Immunocompromised & 3.3 \\
\hline & \\
& \\
&
\end{tabular}

West and Southeast Mexico. As in the overall analyzed group, hypertension, obesity and diabetes were the most frequent underlying comorbidities in the three regions when they were independently analyzed. However, differences in the comorbidities frequencies were found when regions were compared. Hypertension was most frequently found in West (45\%), followed by Northwest (37\%) and Southeast (29\%). Obesity was around 30\% in Northwest and West whereas an $18 \%$ was reported in Southeast. Diabetes was most common in West (34\%) followed by Northwest (22\%) and Southeast (13\%). This data underscores common frequencies in comorbidities in Northwest and West Mexico relative to Southeast (Fig. 2). This might be related to the highest mortality rate in Northwest (31\%) and West (37\%) when compared to Southeast (3\%) (Fig. 2).

\section{Symptoms in hospitalized Mexican patients}

According to the overall weighted average for symptoms, and as reported worldwide [13], the most common symptoms were fever $(80.8 \%)$ followed by cough $(79.8 \%)$, headache (cephalea; 66\%), dyspnea (71.1\%), myalgia 

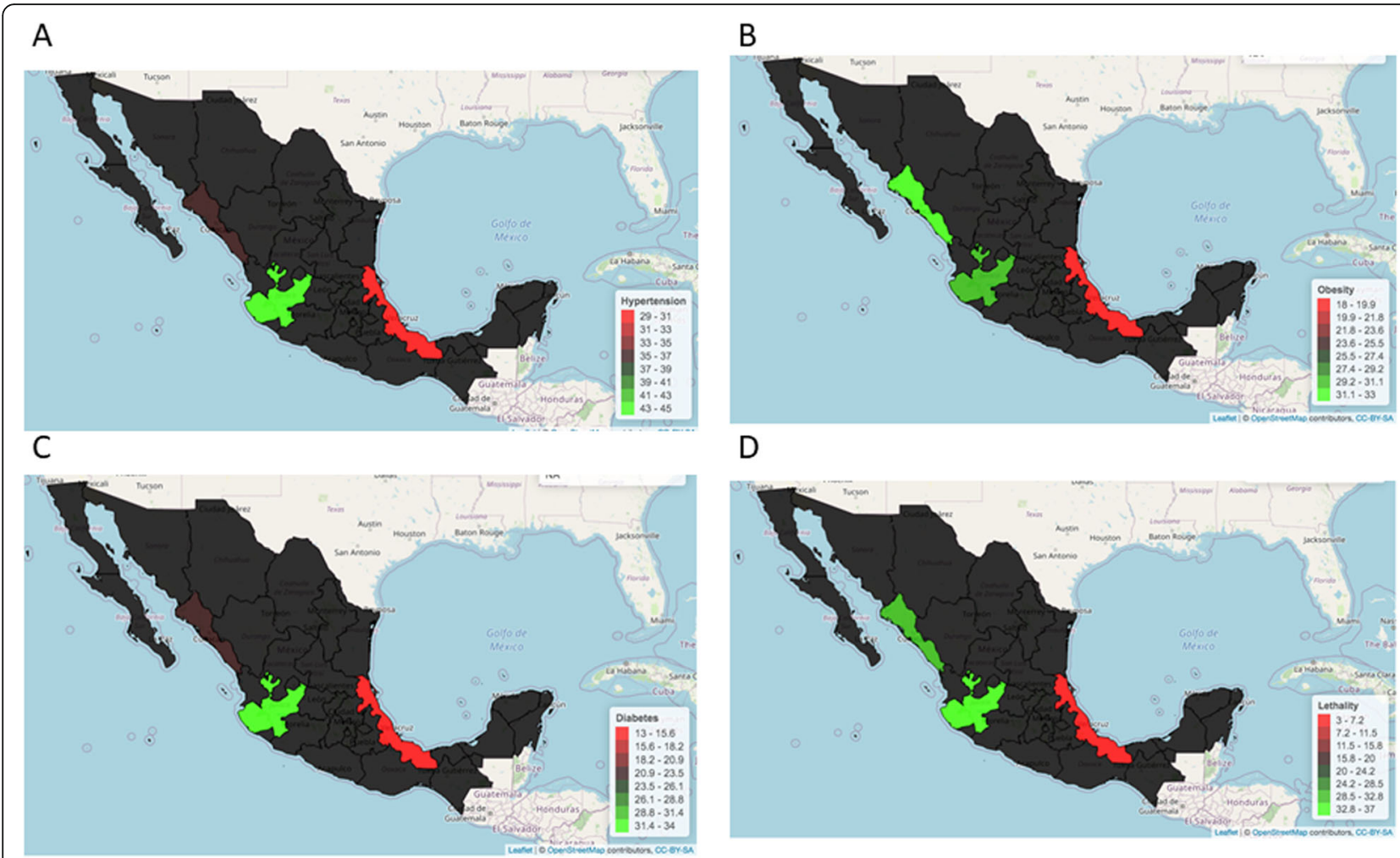

D

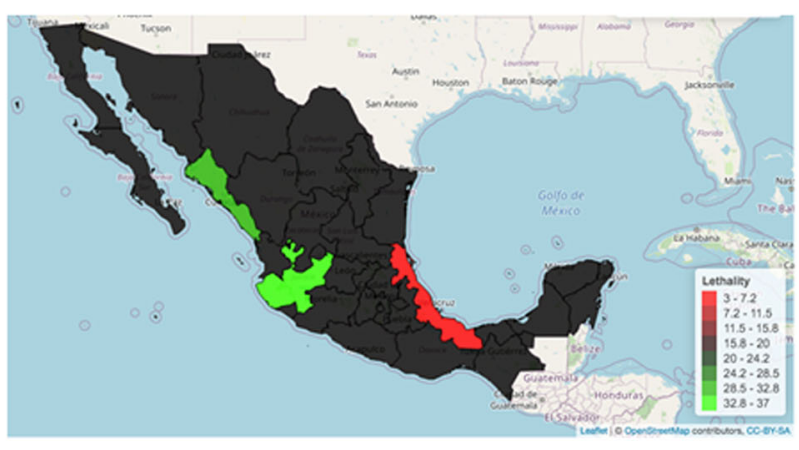

Fig. 2 Differential frequencies in hypertension (A), obesity (B), diabetes (C) and mortality (D) in Northwest, West and Southeast COVID-19 Mexican Patients (figure is our own work)

(53.8\%), joint pain (50.8\%) and odynophagia (34.8\%) (Table 1). As previously reported in South Mexico [11], overall weighted average analysis underscored that diarrhea was overall the main gastrointestinal symptom (21.3\%), followed by abdominal pain (18\%), nausea and vomiting (4.5\%). The prevalence of diarrhea and abdominal pain was similar to a previous report from a meta-analysis including data from patients in the United States [14]. Anosmia (5.9\%) and ageusia (6.1\%) were also found in our analyzed population (Table 1). In Addition, symptoms analysis was performed using weighted method (Suppl. Table 1).

Table 2 COVID-19-associated symptoms in hospitalized patients from distinct regions of Mexico

\begin{tabular}{llll}
\hline Symptoms/Region (\%) & Northwest $(\boldsymbol{n}=\mathbf{1 9 2})$ & West $(\boldsymbol{n}=\mathbf{1 3 0 3})$ & Southeast $(\boldsymbol{n}=\mathbf{1 1 2})$ \\
\hline Headache (\%) & 74.8 & 65.4 & 58.9 \\
Cough (\%) & 83.8 & 79.2 & 80.3 \\
Myalgias (\%) & 29.1 & 56.4 & 66.9 \\
Fever (\%) & 84.4 & 79.8 & 86.6 \\
Odynophagia (\%) & 15.6 & 38 & 31.2 \\
Anosmia (\%) & $\mathrm{NR}$ & 5.9 & 7.1 \\
Dyspnea (\%) & 65.1 & 75.9 & 25.6 \\
Ageusia (\%) & $\mathrm{NR}$ & 6.1 & 7.1 \\
Thoracic pain (\%) & 5.7 & 38.9 & 15.1 \\
Tachypnea (\%) & 16.6 & 7.5 & $\mathrm{NR}$ \\
Cyanosis (\%) & 2 & 7.5 & $\mathrm{NR}$ \\
Nasal congestion (\%) & 10.4 & $\mathrm{NR}$ & $\mathrm{NR}$ \\
Joint pain (\%) & 22.9 & 55 & $\mathrm{NR}$ \\
Fatigue (\%) & 56.2 & $\mathrm{NR}$ & $\mathrm{NR}$ \\
\hline
\end{tabular}


In our analysis, fever, cough and headache were equally distributed between regions. Myalgia was less common in North (29.1\%) relative to West (56.4\%) and Southeast (66.9\%). Odynophagia was most common in West (38\%) and Southeast (31.2\%) than in Northwest (15.6\%). Thoracic pain was more common in West (38.9\%), followed by Southeast (15.1\%) and Northwest (5.7\%). Dyspnea was more common in West (75.9\%), followed by Northwest (65.1\%) and Southeast (25.6\%) (Table 2).

\section{Gastrointestinal symptoms were differentially distributed in distinct regions}

Reported gastrointestinal (GI) symptoms were differentially distributed among COVID-19 patients in distinct regions of Mexico. Diarrhea was the main GI symptom in the analyzed regions. However, its frequency was different across regions. Diarrhea and abdominal pain were more common in West (23.1 and 21\%), followed by Southeast (17.8, and 9.8\%) and Northwest (11.4 and $3.1 \%)$. Nausea and vomiting were more frequently found in Southeast (7.1\%) than in Northwest $(3.1 \%)$, and they were not reported in West (Table 3). We also did the gastrointestinal analysis using weighted method (suppl. Table 2).

\section{Discussion}

According to the evolving data compiled by Johns Hopkins University [3], Mexico is one the top 10 countries in the world with the highest number of COVID-19 associated deaths (accessed on Sep. 22nd, 2020). Underlying comorbidities including metabolic syndrome diseases such as diabetes, hypertension, obesity and cardiovascular diseases represent unfavorable factors for positive COVID-19 outcome. Indeed, from a cohort of 177,133 confirmed COVID-19 cases in Mexico, BelloChavolla et al. recently reported that obesity mediates around 50\% of the effect of diabetes on COVID-19 mortality [15] and in an independent study of 212,802 confirmed SARS-CoV-2 cases in Mexico, HernandezGaldamez et al. reported that noncommunicable diseases underlying comorbidities increase the risk of severe disease [16]. As such, Mexico is currently in the middle of two major public health threats: obesity and SARS-Cov2 infection. According to the Ministry of Health in Mexico, chronic diseases represent 7 of the 10 main causes of death. The most relevant are diabetes and heart disease, both closely related to obesity. The national prevalence of diabetes and hypertension in Mexico are 10.3 and $18.4 \%$, respectively and the prevalence of overweight and obesity in adults older than 20 years old is $75.2 \%$. Obesity, alone, in older than 20 years old accounts for $36.1 \%$ [2].

In this retrospective cohort study, we analyzed data from COVID-19 patients from Northwest, West and Southeast Mexico. Globally, a high mortality rate of $33.1 \%$ was observed. This may be related to the fact that only data from hospitalized patients were analyzed in the present study. It is also important to note that COVID-19-related hospitalization protocols in Mexico prioritize the admission of severe cases. This high mortality is in agreement with current reports puting Mexico among countries with the highest mortality rates associated to COVID-19 worldwide [3]. Since the beginning of the pandemic, the mortality rate reported by the Ministry of Health in the country ranged from 10 to $12 \%$ (including hospitalized and ambulatory patients), still a higher rate than what is reported worldwide (3-4\%). Thus, our finding stress the need to improve strategies to get better outcomes in Mexican population.

Interestingly, our data showed a high mortality rate in Northwest and Western regions relative to Southeast. These discrepancies might be related to the differences in the frequencies of hypertension, obesity and diabetes. These comorbidities were more frequently found in Northwest (37, 30 and 22\%, respectively) and West (45, 30 and 34\%, respectively) relative to Southeast Mexico (29, 18 and 13\%, respectively). The Ministry of Health data however reports that the prevalence of hypertension in Jalisco (West Mexico) as 14\%, in Sinaloa (Northwest) as $16.2 \%$, and in Veracruz (Southeast) as $23.6 \%$. Obesity prevalence as reported by the Ministry of Health in Jalisco, Sinaloa, and Veracruz is $12.9,15.8$, and $22.9 \%$, and the Diabetes prevalence in these states is 7.6, 10.7, and $11.9 \%$, respectively [17]. The differences in the frequencies of comorbidities in our study compared to the data reported by the Ministry of Health might be related to the fact that official data include general population and a large number of individuals when compared to our study population. Moreover, the differences in mortality and comorbidities when regions were independently analyzed in our study may partially be attributed to differences in socio-economic conditions and quality of care, given patients attending to a private hospital in

Table 3 COVID-19-associated gastrointestinal symptoms in hospitalized patients from distinct regions of Mexico

\begin{tabular}{llll}
\hline Symptoms/Region (\%) & Northwest $(\mathbf{n}=\mathbf{1 9 2})$ & West $(\mathbf{n}=\mathbf{1 3 0 3})$ & Southeast $(\mathbf{n}=\mathbf{1 1 2})$ \\
\hline Abdominal pain (\%) & 3.1 & 21 & 9.8 \\
Diarrhea (\%) & 11.4 & 23.1 & 17.8 \\
Nausea and vomiting (\%) & 3.1 & NR & 7.1 \\
\hline
\end{tabular}


Southeast Mexico showed lower mortality rate and reduced frequency in comorbidities relative to patients either attending to public hospitals (Northwest) or hospitals serving state formal workers (West).

According to indicators published in 2018 that include education levels, access to health services, access to social security, access to food, quality of housing spaces and access to basic services in housing, the National Council to the Evaluation of Social Policy [18] estimated that $41.9 \%$ of the population in Mexico is in a situation of poverty. Therefore, it is necessary to prioritize health care services to serve the most vulnerable populations including those in conditions of poverty.

As reported worldwide, fever, cough and headache were the most common symptoms in the cohort. These symptoms were equally distributed between regions. However, slight differences in the frequencies of myalgias and odinophagia were found, with less reported cases in Northwest compared to West and Southeast. Overall, diarrhea was the main gastrointestinal symptom; anosmia and ageusia were also reported. Interestingly, subgroup analyses underscored differences in the frequencies of gastrointestinal symptoms when regions were independently analyzed. Diarrhea and abdominal pain were more common in West, followed by Southeast and Northwest. Taking into account that data from Northwest corresponded to patients attending public hospitals, differences in gastrointestinal symptoms do not seem to be associated with socioeconomic status. However, a larger number of cases need to be analyzed in order to demonstrate this possibility. Moreover, additional factors should be considered to explain the differences in the distribution of gastrointestinal symptoms between regions. In this sense, as in most Latin American countries, the genetic structure of the Mexican population is an admixture of three paternal lineages consisting of Amerindian, European and African ancestry [7]. European and Amerindian ancestry prevail in North and West Mexico, while African and Amerindians prevail in East Mexico. In addition, there are still nearly 15 million Native Amerindians who still maintain their inherited traditions, living across the country [19]. Taking into account that factors including host genetics may affect infectious disease behavior [20], the differences in gastrointestinal symptoms may be related to ethnic composition. Genetic background may also be related to differences in mortality and comorbidities when regions were independently analyzed. Thus, detailed analysis of COVID-19 epidemiologic status in a large number of regions with similar genetic background and different socio-economic status is required to assess the roles these confounders play in observed symptoms and outcomes.

Limitation of our study include: 1) overall sample size, 2) distinct sample size between regions, 3) possible selection bias given that data correspond to patients from public and private hospitals in distinct proportions, 4) possible selection bias given the protocols in Mexico prioritize the hospitalization of severe cases.

\section{Conclusion}

The emergency imposed by COVID-19 pandemic highlights the need for better notification and recording systems to estimate the real impact of the disease behavior globally. Close monitoring of underlying comorbidities, symptoms and outcomes is recommended in the construction of predictive models to determine risk populations for infection and poor outcomes. Joint efforts in low-income countries as in most of Latin America are needed to assess the disease behavior and to lower its impact. Given similar economic, socio-demographic, environmental and ethnic conditions prevail in Latin America, our findings may benefit the entire region. However, future studies are required to determine the specific strategies to handle the pandemic at local levels.

\section{Abbreviations}

ACE2: Angiotensin Receptor 2; Cl: Confidence Interval; COVID-19: Coronavirus Disease-19; Gl: Gastrointestinal; RT-PCR: Real-time Polymerase Chain Reaction; SARS-CoV-2: Severe Acute Respiratory Syndrome Coronavirus 2

\section{Supplementary Information}

The online version contains supplementary material available at https://doi. org/10.1186/s12879-021-06252-y.

Additional file 1 Suppl. Table 1: Symptoms analysis was performed using weighted method. Suppl. Table 2. Gastrointestinal analysis was performed using weighted method.

\section{Acknowledgements}

We would like to thank Drs. Villagrán-Olivas and Remes-Troche and their colleagues for contribution with their published data Ref\#10-11. We thank all health care personnel in this pandemic era.

\section{Authors' contributions}

Study concept and design (HA), acquisition of data (NF, ECV, MHS, AP), analysis and interpretation of data $(\mathrm{HA}, \mathrm{HB}, \mathrm{GO})$, drafting of manuscript ( $\mathrm{HA}$, $H B, A P, N F)$, statistical analysis (GO), obtained funding (HA), material support $(H A, H B)$. All authors have read and approved the manuscript.

\section{Funding}

This project was supported (in part) by the National Institute on Minority Health and Health Disparities of the National Institutes of Health under Award Number G12MD007597. The funders had no role in study design, data collection and analysis, decision to publish, or preparation of the manuscript.

Availability of data and materials

All weighted de-identified data used here is included in this study.

\section{Declarations}

Ethics approval and consent to participate

The de-identified data in the study does not need consent form since it is available in the relative published articles or from the ministry of health. All resources have been cited in the paper. 


\section{Consent for publication}

Not applicable.

\section{Competing interests}

No potential conflicts of interest are disclosed.

\section{Author details}

'La Universidad del Zulia, Faculty of Medicine, School of Medicine, Maracaibo, Zulia state 4002, Venezuela. ${ }^{2}$ Department of Immunology, Institute of Biomedical Research, National Autonomous University of Mexico, Ciudad University, CP 04510 Mexico City, Mexico. ${ }^{3}$ Mexican Social Security Institute, Guadalajara, Mexico. ${ }^{4}$ College of Engineering and Technology, American University of the Middle East, Egaila, Kuwait. ${ }^{5}$ Department of Medicine, Department of Pathology and Cancer Center, Department of Biochemistry \& Molecular Biology, Howard University College of Medicine, 2041 Georgia Avenue, N.W, Washington, D.C 20060, USA.

Received: 6 December 2020 Accepted: 28 May 2021

Published online: 11 June 2021

\section{References}

1. Odometer $\mathbf{W}$. The cases/deaths of Covid-19 for South America plus North America; 2020.

2. Secretary of Health of Mexico, National Institute of Statistics and Geography, National Institute of Public Health. National Health and Nutrition Survey 2018. 2019.

3. Johns Hopkins University. COVID-19 Dashboard by the Center for Systems Science and Engineering (CSSE) at Johns Hopkins University (JHU) 2020 [cited 2020; Available from: https://coronavirus.jhu.edu/map.html.

4. Grant MC, Geoghegan L, Arbyn M, Mohammed Z, McGuinness L, Clarke EL, et al. The prevalence of symptoms in 24,410 adults infected by the novel coronavirus (SARS-CoV-2; COVID-19): a systematic review and meta-analysis of 148 studies from 9 countries. PLoS One. 2020;15(6):e0234765. https://doi. org/10.1371/journal.pone.0234765.

5. Gavriatopoulou M, Korompoki E, Fotiou D, Ntanasis-Stathopoulos I, Psaltopoulou T, Kastritis E, et al. Organ-specific manifestations of COVID-19 infection. Clin Exp Med. 2020;20(4):493-506. https://doi.org/10.1007/s10238020-00648-x.

6. Burgner D, Jamieson SE, Blackwell JM. Genetic susceptibility to infectious diseases: big is beautiful, but will bigger be even better? Lancet Infect Dis. 2006;6(10):653-63. https://doi.org/10.1016/S1473-3099(06)70601-6.

7. Ruiz-Linares A, Adhikari K, Acuña-Alonzo V, Quinto-Sanchez M, Jaramillo C, Arias W, et al. Admixture in Latin America: geographic structure, phenotypic diversity and self-perception of ancestry based on 7,342 individuals. PLoS Genet. 2014;10(9):e1004572. https://doi.org/10.1371/journal.pgen.1004572.

8. Martinez-Cortes $\mathrm{G}$, et al. Admixture and population structure in Mexicanmestizos based on paternal lineages. J Hum Genet. 2012;57(9):568-74. https://doi.org/10.1038/jhg.2012.67.

9. Ministry of Health Mexico, General Directorate of Health Information. 2020.

10. Villagrán-Olivas KA, Alejandro T-ZL, Adolfo E-G. Clinical-epidemiological characteristics of COVID-19 patients in a Hospital of Sinaloa, México. REVM EDUAS. 2020;10:65-79.

11. Remes-Troche JM, Ramos-de-la-Medina A, Manríquez-Reyes M, MartínezPérez-Maldonado L, Lara EL, Solís-González MA. Initial gastrointestinal manifestations in patients with severe acute respiratory syndrome coronavirus 2 infection in 112 patients from Veracruz in southeastern Mexico. Gastroenterology. 2020;159(3):1179-81. https://doi.org/10.1053/j.ga stro.2020.05.055

12. Giannouchos TV, et al. Characteristics and risk factors for COVID-19 diagnosis and adverse outcomes in Mexico: an analysis of 89,756 laboratoryconfirmed COVID-19 cases. Eur Respir J. 2020.

13. Garibaldi BT, et al. Patient Trajectories Among Persons Hospitalized for COVID-19: A Cohort Study. Ann Intern Med. 2020.

14. Nobel YR, Phipps M, Zucker J, Lebwohl B, Wang TC, Sobieszczyk ME, et al. Gastrointestinal symptoms and coronavirus disease 2019: a case-control study from the United States. Gastroenterology. 2020;159(1):373-5 e2. https://doi.org/10.1053/j.gastro.2020.04.017.

15. Shamseer $L$, Moher D, Clarke M, et al. Preferred reporting items for systematic review and meta-analysis protocols (PRISMA-P) 2015: elaboration and explanation BMJ. 2015;350:1-25.
16. Hernandez-Galdamez DR, et al. Increased risk of hospitalization and death in patients with COVID-19 and pre-existing noncommunicable diseases and modifiable risk factors in Mexico. Arch Med Res. 2020;51(7):683-9. https:// doi.org/10.1016/j.arcmed.2020.07.003.

17. Report, N.H.a.N.S.R., Presentation of results National Health and Nutrition Survey Results Report - 2020.

18. Policy, N.E.C.o.t.S.D., Vulnerable and historically discriminatory groups in the context of the sanitary emergency caused by the SARS-CoV-2 virus (COVID2019). 2020.

19. Security. MloS: Interim algorithms for the care of COVID-19. 2020.

20. Ovsyannikova IG, Haralambieva IH, Crooke SN, Poland GA, Kennedy RB. The role of host genetics in the immune response to SARS-CoV-2 and COVID-19 susceptibility and severity. Immunol Rev. 2020;296(1):205-19. https://doi. org/10.1111/imr.12897.

\section{Publisher's Note}

Springer Nature remains neutral with regard to jurisdictional claims in published maps and institutional affiliations.
Ready to submit your research? Choose BMC and benefit from:

- fast, convenient online submission

- thorough peer review by experienced researchers in your field

- rapid publication on acceptance

- support for research data, including large and complex data types

- gold Open Access which fosters wider collaboration and increased citations

- maximum visibility for your research: over $100 \mathrm{M}$ website views per year

At $\mathrm{BMC}$, research is always in progress.

Learn more biomedcentral.com/submissions 